\title{
MORPHOLOGIC ALTERATIONS OF MYOCARDIUM UNDER CONDITIONS OF LEFT VENTRICULAR ASSISTANCE
}

Christophe Depre, MD, PhD, Xavier Havaux, BSc, Robert Dion, MD, and Jean L. Vanoverschelde, MD, PhD, Brussels, Belgium

Implantation of a left ventricular assist device (LVAD) is used as a bridge for patients expecting heart transplantation. By changing the loading conditions of the failing heart, the LVAD most probably alters heart structure. We report the pathologic examination of myocardium from a patient before and after 4 months of LVAD use. We show that unloading the heart with the LVAD leads to a dedifferentiation process of the cardiomyocytes comparable to that found in "hibernating" myocardium.

Clinical summary. An 18-year-old male patient was seen for idiopathic dilated cardiomyopathy that did not improve with maximal drug therapy. Because of the unavailability of a compatible heart donor, the patient was surgically treated with partial left ventricular resection (resection of the anterolateral wall and correction of the mitral regurgitation). The lack of postoperative improvement led to the insertion of an implantable LVAD (Novacor; Baxter Healthcare Corp., Novacor Div., Oak-

From the Divisions of Cardiology and Cardiovascular Surgery, University of Louvain Medical School, Brussels, Belgium.

Received for publication July 28, 1997; accepted for publication August 27, 1997.

Address for reprints: Christophe Depre, MD, PhD, Division of Cardiology, University of Texas Medical School, 6431 Fannin, MSB 1.608, Houston, TX 77030.

J Thorac Cardiovasc Surg 1998;115:478-9

Copyright (C) 1998 by Mosby, Inc.

0022-5223/98 $\$ 5.00+0 \quad \mathbf{1 2 / 5 4 / 8 5 6 7 7}$

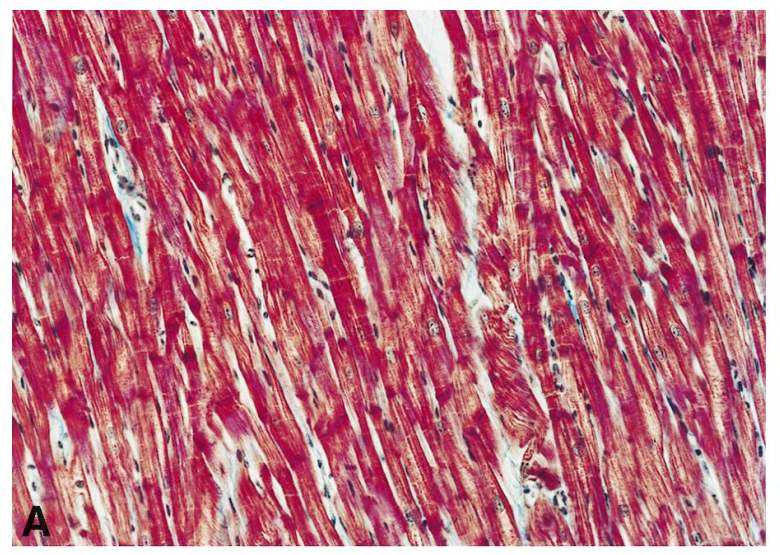

land, Calif.). After 4 months of stabilization, the device was removed. Three months later, a heart donor was found and the patient underwent transplantation. Myocardial tissues retrieved during partial left ventricular resection, at explantation of the LVAD, and at transplantation were kept for pathologic examination. All tissue fragments were embedded in paraffin, cut in $7 \mu \mathrm{m}$ thick sections, then stained with Masson trichrome stain and periodic acid-Schiff reaction.

Results. Morphologic characteristics of the fragments retrieved during partial left ventricular resection were not different from normal tissue (Fig. 1, $A$ ). The cardiomyocytes covered $90 \%$ of the total surface, whereas extracellular matrix covered about 7\%. Cardiomyocytes were globally normal in appearance on light-microscopic examination, with cytosol filled with myofilaments and nuclei with normal chromatin content (Fig. 1, $A$ ). About $15 \%$ of the cardiomyocytes showed slight accumulation of glycogen in the cytosol (Fig. 2, A). After 4 months with LVAD, morphologic inspection of the myocardial tissue revealed major differences (Figs. 1 and 2). The cellular volume was increased compared with the previous fragments. The rod-shaped cells found initially were now potato-like in appearance, dilated and spherical (Fig. 2, A). Their myofilament content was dramatically decreased, and their nuclei were condensed and pyknotic (Fig. 1, B). The percentage of surface covered by extracellular matrix was increased to $13 \%$ (Fig. 1, B). About $95 \%$ of the cardiomyocytes showed an intracellular accumulation of glycogen (Fig. 2, B). In the fragments retrieved during trans-

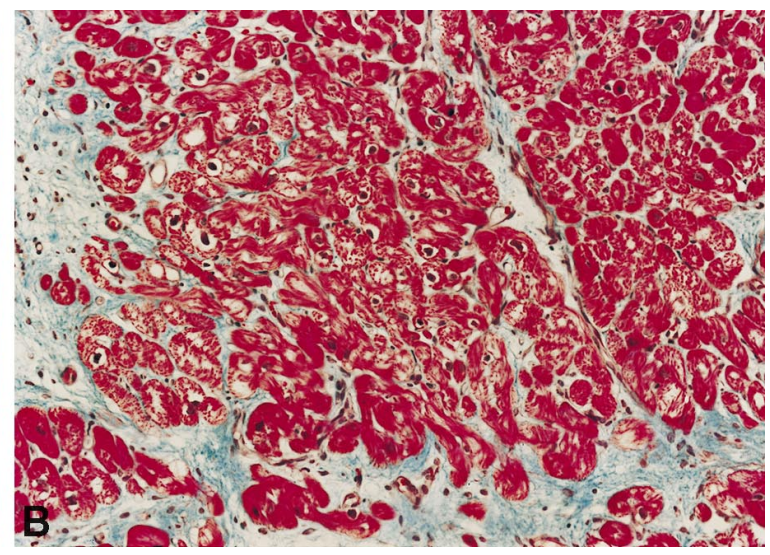

Fig. 1. Morphologic appearance of myocardium before and after LVAD implantation. The myofilament content is normal before LVAD implantation (A), whereas it is reduced after 4 months of LVAD support (B). The nuclei are normal before and pyknotic 4 months after LVAD implantation (Masson's trichrome; original magnification $\times 20$.) 

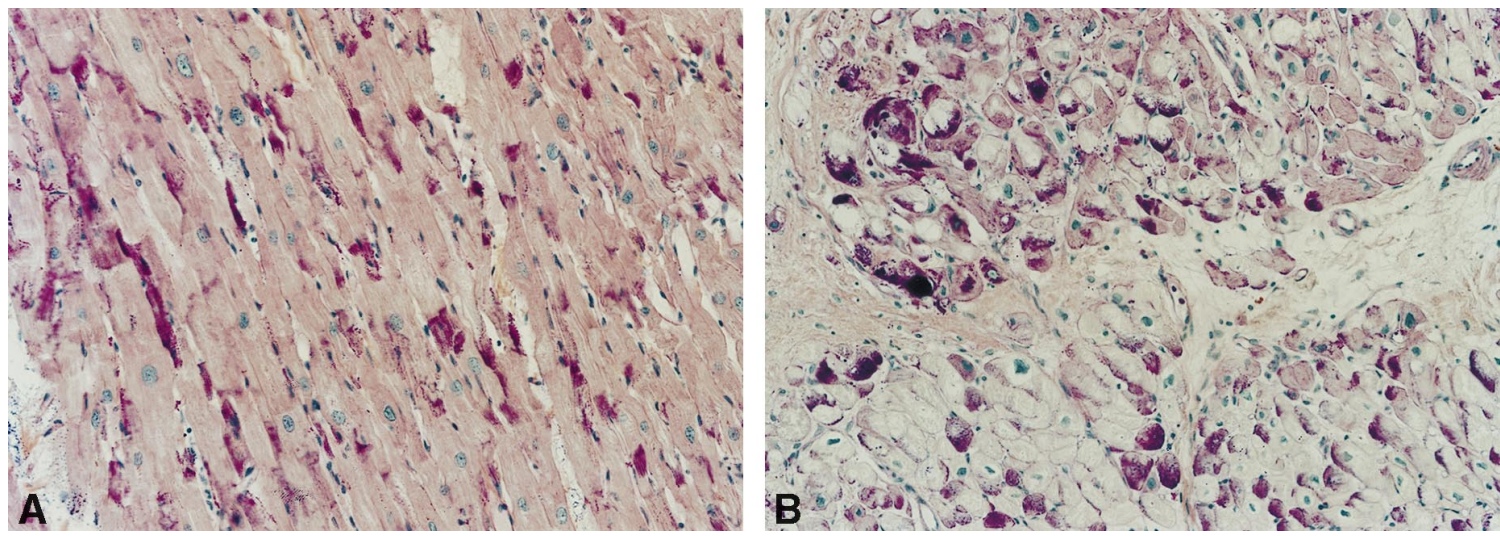

Fig. 2. Glycogen accumulation in cardiomyocytes. A, A few cardiomyocytes show an intracellular accumulation of dark-red material compatible with glycogen. After 4 months of LVAD support, most cardiomyocytes display an important accumulation of glycogen in their cytosol. B, Periodic acid-Schiff reaction (original magnification $\times 20$.)

plantation, the cellular morphologic characteristics had returned to a normal pattern in most of the cells, but the percentage of intercellular fibrosis remained increased.

Discussion. Our data thus show that heart structure may create important—albeit reversible—morphologic alterations after several months of LVAD implantation. These alterations are mainly a loss of myofilaments, an intracellular accumulation of glycogen, and an increase in extracellular matrix. The alterations that we found in this case are similar to those previously described in human "hibernating" myocardium. ${ }^{1,2}$ Patients with hibernating myocardium in whom such morphologic alterations are found have functional improvement after revascularization, ${ }^{3}$ suggesting that these phenotypic alterations are potentially reversible. Such a phenotypic shift is still poorly understood. There is accumulating evidence that the structural changes observed are the consequence of a reverse differentiation process, because these altered cardiomyocytes are similar to fetal cardiomyocytes. ${ }^{4}$ It remains unknown, however, through what mechanisms unloading the heart with the LVAD induces such alterations and whether this adaptation is a prerequisite for subsequent functional improvement.

\section{REFERENCES}

1. Vanoverschelde JL, Wijns W, Depre C, et al. Mechanisms of chronic regional post-ischemic dysfunction in humans: new insights from the study of non-infarcted collateral-dependent myocardium. Circulation 1993;87:1513-23.

2. Depre C, Vanoverschelde JL, Melin JA, et al. Structural and metabolic correlates of the reversibility of chronic left ventricular ischemic dysfunction in humans. Am J Physiol 1995;268 H1265-75.

3. Depre C, Vanoverschelde JL, Gerber B, Borgers M, Melin JA, Dion R. Correlation of functional prognosis with myocardial morphology and metabolism in patients undergoing coronary bypass surgery. J Thorac Cardiovasc Surg 1997;113:371-8

4. Ausma J, Schaart G, Thone F, et al. Chronic ischemic viable myocardium in man: aspects of dedifferentiation. Cardiovasc Pathol 1995;4:29-37. 